\title{
Proteomic analysis of human cerebral cortex in epileptic patients
}

\author{
Jong-Pil Eun ${ }^{2}$, Ha-Young Choi \\ and Yong-Geun Kwak ${ }^{1,3}$ \\ ${ }^{1}$ Deparment of Pharmacology \\ ${ }^{2}$ Deparment of Neurosurgery Institute of Cardiovascular Research \\ Chonbuk National University Medical School \\ Chonju 560-182, Korea \\ ${ }^{3}$ Corresponding author: Tel, 82-63-270-3091; \\ Fax, 82-63-275-2855; E-mail, ygkwak@chonbuk.ac.kr
}

Accepted 14 April 2004

Abbreviations: 2-DE, 2-dimensional electrophoresis; ESI-Q-TOF, electrospray ionization quadupole time of flight; GPDH, Glycerol phosphate dehydrogenase; GPX, glutathione peroxidase; MALDITOF, matrix-assisted laser desorption/ionization-time-of flight; SOD, superoxide dismutase

\begin{abstract}
Epilepsy affects more than $0.5 \%$ of the world population and is known to be associated with a large genetic component eliciting an electrical hyperexcitability in the central nervous system. However, its pathogenic mechanisms remain poorly understood. In order to gain greater molecular incite in the pathogenesis in epilepsy, we analyzed proteomes of human cerebral cortices. Quantitative proteome analysis was used to compare signals corresponding to individual proteins between epileptic cerebral cortices from patients with temporal lobe epilepsy and age-matched non-epileptic subjects. To minimize individual variations, gender and age of the patients were matched. Changes of several spots were consistent among 6 pairs of epileptic patients and nonepileptic subjects. One of the spots was identified as the mitochondrial type $M n$-superoxide dismutase (Mn-SOD) confirmed by Western blot analysis with Mn-SOD antibody and enzyme activity assay. Such results were agreeable with chemical and physical parameters given by the 2-dimensional electrophoresis (2-DE) gel. Mn-SOD was consistently down-regulated in epileptic cerebral cortices compared with those of nonepileptic subjects. Our results demonstrate a clear link between pathogenesis of epilepsy and SOD. Additionally, we identified four proteins that were consistently over-expressed in
\end{abstract}

all epileptic temporal neocortices specimens and the other four proteins that were found to be expressed less than non-epileptic control subjects. These proteomic data provide cellular markers in the understanding mechanism of the epilepsy pathogenesis.

Keywords: cerebral cortex; epilepsy; proteomics; superoxide dismutase; temporal lobe

\section{Introducton}

Epilepsy is a neuronal disease characterized by recurrent seizures, caused by a non-controlled discharge (hyperexcitability) of neurons of either the whole cortex (generalized epilepsies) or localized brain areas (partial epilepsies). In developed countries, the age adjusted incidence of recurrent unprovoked seizures ranges from 24 to 53 out of 1000 persons per year, and it is consistent across geographical areas. The age adjusted prevalence rate varies from 4 to 8 per 1,000 (Stefan et al., 2001). Epilepsy is a common and heterogeneous neurological disorder arising from biochemical and molecular events that are yet to be fully understood. The development of molecular markers and genomic resources has facilitated the isolation of genes responsible for rare monogenic epilepsies in human and mouse. Many of the identified genes encode ion channels such as $\mathrm{Na}^{+}, \mathrm{K}^{+}$and $\mathrm{Ca}^{2+}$ channels or other components of neuronal signaling (Meisler et al., 2001; Park et al., 2003). Although the reports are not divergent (Oliver et al., 1990; Mori and Yokoyama, 1993; Murashima et al., 1998; Halliwell and Gutteridge, 1999b; Ben-Menachem et al., 2000; Erakovic et al., 2000; Liang et al., 2000; Patel, 2002), oxidative stress is emerging as a mechanism that may play an important role in the etiology of seizure. However, it is remained unclear which types of antioxidant systems are involved.

Despite of a long time study in epilepsy, the progress of understanding its molecular mechanism has been limited. Proteomics is a technique that enables one to find proteins changed by cells' response to internal states (e.g. the cell cycle), external stimulations, or developmental changes. We have analyzed proteins expressed in cerebral cortex of human epileptic temporal lobe using proteomics technique and found consistent decrease of Mn-SOD in temporal neocortex of epileptic patients. In addition, 
the levels of eight other proteins were found to be significantly different between temporal neocortexes from nonepileptic and epileptic subjects.

\section{Materials and Methods}

\section{Sample preparation}

Twenty four subjects were recruited from Chonbuk National University Hospital for this study. Twelve control patients without seizure had the tumor in the temporal lobe. The ages ranged from 27 to 50 yearold. Before extirpation of the tumor, a small piece of intact temporal neocortex in the trajectory for tumor resection was obtained from each patient for control study. Histopathological findings showed seven as glioblastoma multiforme, three as sphenoid ridge meningioma, and two as malignant lymphoma. In contrast, 12 epileptic patients between 27 to 48 years had complex partial seizures. Seizure duration was from 2 to 17 years. Evaluation methods for resection of the epileptogenic zone included chronic video-electroencephalogram monitoring with surface and subdural grid electrodes, magnetic resonance imaging, 3 dimensional surface rendering of magnetic resonance imaging, interictal positron emission tomography, neuropsychological test, and Wada test. Epileptogenic zones of epileptic patients were located in the temporal neocortex. Immediately after temporal lobectomy, a portion of the resected temporal neocortex was obtained for this study of epilepsy group. Histopathological findings revealed neuronal migration disorder of the temporal neocortex in five, gliosis of the temporal neocortex with hippocampal sclerosis in five, and gliosis of the temporal neocortex without hippocampal sclerosis in two. This study was approved by the Ethics Committee of Chonbuk National University, Medical School and the fully informed and written consent was obtained from all subjects.

\section{Two-dimensional electrophoresis}

Tissues were frozen with liquid nitrogen and were ground to fine powders. After lysis buffer containing $8 \mathrm{M}$ urea, 4\% CHAPS, $40 \mathrm{mM}$ Tris base, 1\% DTT andd $0.5 \%$ immobilized $\mathrm{pH}$ gradient (IPG) buffer was added, the sample suspension was sonicated in short bursts on ice. The lysate was centrifuged in a $\mathrm{mi}-$ crocentrifuge at $30,000 \mathrm{~g}$ for $15 \mathrm{~min}$ at $4^{\circ} \mathrm{C}$. The supernatant was stored at $-80^{\circ} \mathrm{C}$ until used. The first dimension of 2-dimensional electrophoresis (2-DE) was performed on a IPG strip (Amersham Biosciences). Linear $\mathrm{pH}$ 3-10 IPG strips $(24 \mathrm{~cm})$ were rehydrated overnight at room temperature in rehydrating buffer (8 M urea, 1\% DTT, 2\% CHAPS, and 0.5\% IPG buffer). Sample of $500 \mathrm{~g}$ was applied during rehydration. The first dimension was run for 53,500 $\mathrm{Vh}$ at $20^{\circ} \mathrm{C}$ using the following conditions: $500 \mathrm{~V}$ for $1 \mathrm{~h}, 1,000 \mathrm{~V}$ for $1 \mathrm{~h}$; and $8,000 \mathrm{~V}$ for $6 \mathrm{~h}$ and 30 min. Next, gels were equilibrated for $30 \mathrm{~min}$ in equilibration buffer I containing $50 \mathrm{mM}$ Tris-Cl, $6 \mathrm{M}$ urea, $30 \%$ glycerol, $2 \%$ sodium dodesyl sulfate (SDS) and $0.1 \%$ DTT and equilibration buffer II containing $50 \mathrm{mM}$ Tris-Cl, $6 \mathrm{M}$ urea, $30 \%$ glycerol, $2 \%$ SDS and $0.25 \%$ iodoacetic acid. The second dimension was run on Ettan DALT II system (Amersham Biosciences). A $12.5 \%$ SDS-polyacrylamide slab gel was used for the second dimension gel electrophoresis. The IPG strips were placed on the surface of the second dimension gel, and then were sealed with $0.5 \%$ Agarose in SDS electrophresis buffer containing $25 \mathrm{mM}$ Tris base, $192 \mathrm{mM}$ glycine and $0.1 \%$ SDS. The gels were placed into Ettan DALT II system chamber containing $1 \times$ SDS electrophoresis buffer. The gels were run overnight at $110 \mathrm{~V}$ until the dye front reached the bottom of the gel.

\section{Silver staining}

Silver staining of gels was performed as modified silver staining protocol using Silver Stain PlusOne kit (Amersham Biosciences). The use of glutaraldehyde in the sensitization step and formaldehyde in the silver impregnation step was omitted. After electrophoresis, the gels were fixed with $40 \%$ methanol and $10 \%$ acetic acid for $30 \mathrm{~min}$. The gels were sensitized by incubating in sensitizing solution $(0.2 \%$ sodium thiosulphate, $30 \%$ methanol, sodium acetate, $68 \mathrm{~g} / \mathrm{L})$, and it was then rinsed with three changes of distilled water for $5 \mathrm{~min}$ each. After rinsing, the gels were incubated in $0.25 \%$ silver nitrate for $20 \mathrm{~min}$. After incubation, the silver nitrate was discarded, and the gels were rinsed twice with distilled water for $1 \mathrm{~min}$ and then developed in $0.15 \%$ formaldehyde in $2.5 \%$ sodium carbonate with intensive shaking. After the desired intensity of staining was achieved, the development was terminated with $1.46 \%$ EDTA.

\section{Image analysis}

The silver-stained 2-DE gels were scanned with LabScan software on Imagescanner (Amersham Biosciences), and digitized and analyzed using ImageMaster 2D (Amersham Biosciences). Matching of the spots was performed by use of a reference gel prepared from six gels. Spot standardization was carried out for all matched spots.

\section{Destaining}

Silver-stained proteins were destained with chemical reducers to remove the silver as described previously with following critical modifications (Scheler et al., 
1998). Potassium ferricyanide and sodium thiosulfate were prepared as two stock solutions of $30 \mathrm{mM}$ potassium ferricyanide and $100 \mathrm{mM}$ sodium thiosulfate, which were dissolved in water. A working solution was prepared by mixing a $1: 1$ ratio prior to use. After interesting protein spots were excised from the gel, thirty to fifty microliters of working solution were added to cover the gels and occasionally vortexed. The stain intensity was monitored until the brownish color disappeared, then the gel was rinsed a few times with water to stop the reaction. Next, $200 \mathrm{mM}$ ammonium bicarbonate was added to cover the gel for $20 \mathrm{~min}$ and was then discarded. Subsequently, the gel was cut into small pieces, washed with water, and was dehydrated repeatedly with changes of acetonitrile until the gel pieces turned into opaque white color. The gel pieces were dried in a vacuum centrifuge for $30 \mathrm{~min}$.

\section{Trypsin digestion of proteins in-gel}

Enzymatic digestion was performed as previously described (Hellman et al., 1995; Shevchenko et al., 1996). Briefly, digestion was performed with 5-10 ng $/ \mu \mathrm{l}$ of trypsin and $50 \mathrm{mM}$ ammonium bicarbonate and incubated overnight at $37^{\circ} \mathrm{C}$. Following enzymatic digestion, the resultant peptides were extracted three times with $10-20 \mu \mathrm{l}$ of $5 \%$ trifluoroacetic acid in $50 \%$ acetonitrile and dried using a vacuum centrifuge for $30 \mathrm{~min}$.

\section{Identification of proteins}

The dried samples were analyzed by matrix-assisted laser desorption/ionization-time-of flight (MALDI-TOF) mass spectrometer (Voyager-DE PRO) for peptide mass fingerprinting, and by electrospray ionization quadupole time of flight (ESI-Q-TOF) mass spectrometry analysis for peptide sequencing. The database searches were carried out using MS-Fit, which has access to world wide web at http://kr.expasy.org or http://www.ncbi.nlm.nih.gov.

\section{Western blot}

Brain mitochondria were prepared according to Lai and Clark (1979). Proteins $(30 \mu \mathrm{g})$ were separated on $12 \%$ SDS-PAGE and transferred onto polyvinylidene difluoride (PVDF) membranes (Amersham Biosciences) in Tris-glycine buffer ( $\mathrm{pH} 8.4$ ) containing $20 \%$ methanol. The membrane was then blocked in $5 \%$ fat-free dry milk in phosphate-buffered saline/0.1\% Tween-20. After overnight incubation with the antibody specific for Mn-superoxide dismutase (Mn-SOD) (Santa Cruz), the blot was incubated with a horseradish peroxidase-conjugated secondary antibody $(1: 5,000)$. Proteins were visualized using an enhanced chemiluminescence system (Intron) and the LAS $3000 \mathrm{im}$ aging system (Fugi).

\section{SOD enzyme assay}

SOD activity was measured spectrophotometrically using a Bioxytech SOD-525 kit (Oxis Health Products) that is based on the SOD-mediated increase in the rate of autoxidation of $5,6,6 \mathrm{a}, 11 \mathrm{~b}$-tetrahydro-3, 9,10-trihydroxybenzo[c]fluorene in aqueous alkaline solution to yield a chromophore with maximum absorbance at $525 \mathrm{~nm}$. Tissues were homogenized in 50 $\mathrm{mM}$ Tris- $\mathrm{HCl}$ solution and then centrifuged at 14,000 $g$ for 5 min at $4^{\circ} \mathrm{C}$. The supernatants were mixed with ethanol and chloroform (7.5:32.5 v/v) and centrifuged again at $3,000 \mathrm{~g}$ for $5 \mathrm{~min}$ at $4^{\circ} \mathrm{C}$. The supernatants were diluted with SOD-525 kit solution and then SOD activities were measured by detecting absorbance at $525 \mathrm{~nm}$ (Nebot et al., 1993).

\section{Results}

Protein profiles from the temporal neocortex of twelve intractable temporal lobe epileptic patients undergoing

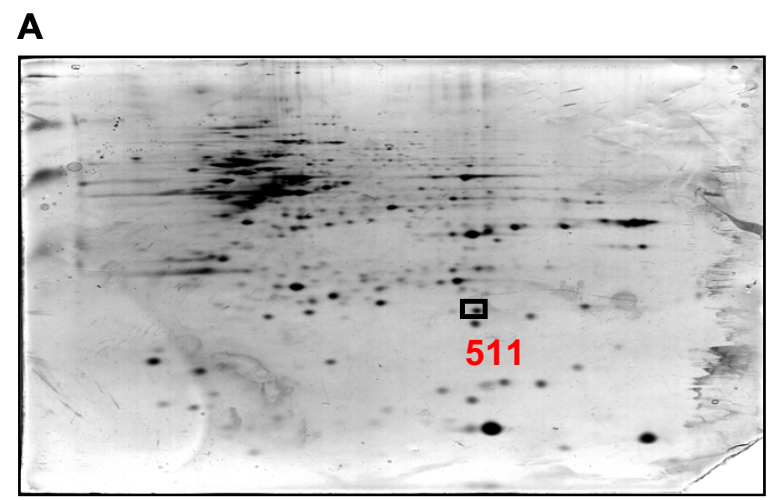

B

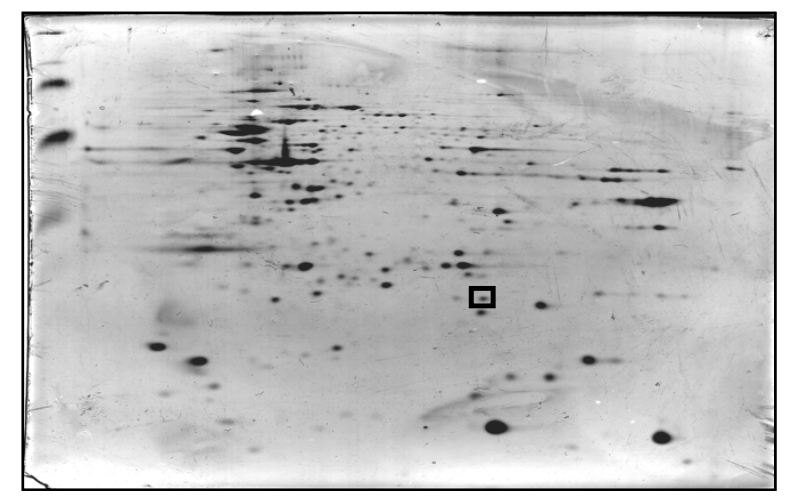

Figure 1. Representative 2-DE gel map derived from the cerebral cortexes of non-epileptic (A) and epileptic (B) human temporal lobes. The gels were visualized with silver staining. One of the proteins expressing differentially between non-epileptic and epileptic cerebral cortexes was marked as a spot number 511 . 
therapeutic resection of the epileptogenic zone and from a small piece of intact temporal neocortex of twelve non-epileptic subjects undergoing trajectory for tumor resection were analyzed by 2-D PAGE and stained with silver. A representation of one set among them is shown in Figure 1 . We routinely observed between $650-800$ spots from protein of $400 \mu \mathrm{g}$ when stained with routine silver staining procedures. Human samples are notorious for their individual variations in protein expression level. Therefore, we matched gender and age of the samples as much as we can.

One of the spots (spot number 511) showed consistent change of its protein level among 6 pairs of epileptic and non-epileptic samples (Figure 1 and 2). Its pl was about 7.0 and its molecular weight was estimated to be $18 \mathrm{kDa}$. The spot was excised and destained. Then it was digested by trypsin overnight and was analyzed by MALDI-TOF MS for peptide finger printing as well as by ESI Q-TOF MS for

\section{A}

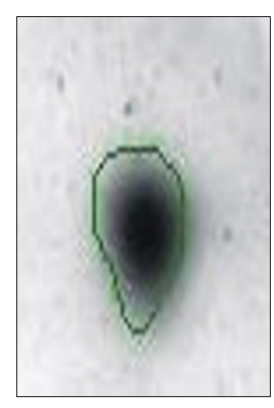

Non-epileptic

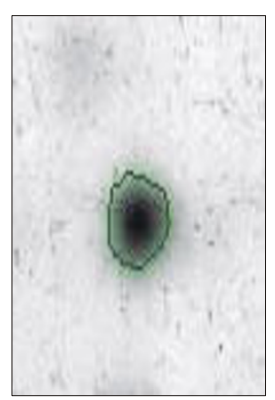

Epileptic
B

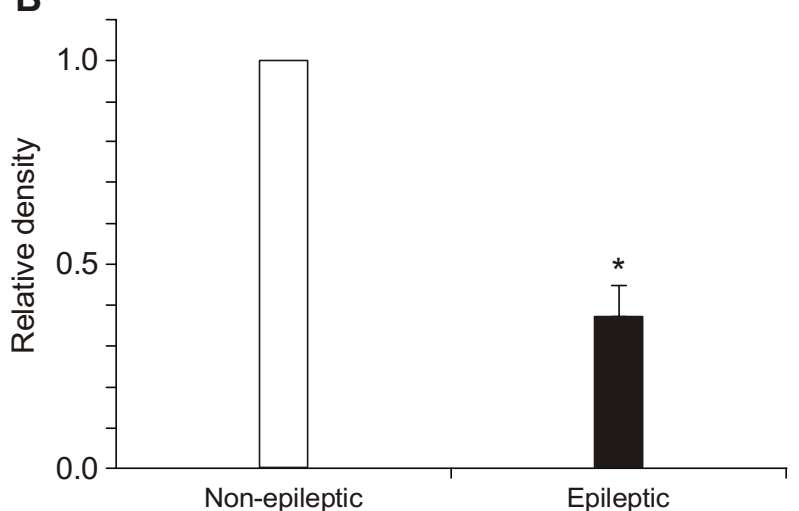

Figure 2. The protein level of spot 511 showed consistent decrease in twelve epileptic human temporal lobes compared with non-epileptic temporal lobes. A, a representative pair of enlarged spot 511 in the gels from non-epileptic and epileptic human temporal lobes. B, The averaged values of the densities for spot 511 in the gels from non-epileptic and epileptic human temporal lobes. Results of densitometric analysis are expressed as mean \pm SEM from 12 experiments $\left({ }^{*} P<0.001\right)$. peptide sequencing. The results of the MALDI-TOF $M S$ and ESI Q-TOF MS indicated unequivocally that the identity of the spot was the mitochondrial Mn-SOD (Figure 3).

To confirm the change of the Mn-SOD enzyme level, we carried out Western blot using antibody against Mn-SOD. The results of the Western blot were consistent with the result of the 2-D electrophoresis gel (Figure 4A). In epileptic cerebral cortex, the protein expression level of $\mathrm{Mn}$-SOD was decreased to $45 \pm 9 \%(n=12)$ of the cerebral cortex from the nonepileptic subjects (Figure 4B).

We also measured the enzyme activity at the tissues. This enzyme assay could not separate the activity of $M n-S O D$ from other types of SOD in the tissue. But it was still interesting that the total SOD activity of the epileptic temporal lobe was consistently decreased to $37 \pm 8 \%(n=6)$ of the non-epileptic temporal lobe (Figure 5).

And also, we found additional eight proteins which were significantly and consistently different between temporal neocortexes from twelve nonepileptic and twelve epileptic subjects on 2-D gels. Figure 6 is a representation of one set among them. The significant spots were also identified by MALDI-TOF MS for peptide finger printing as well as by ESI Q-TOF MS for peptide sequencing (Table 1). Four proteins were identified as being consistently overexpressed in all

\section{A}

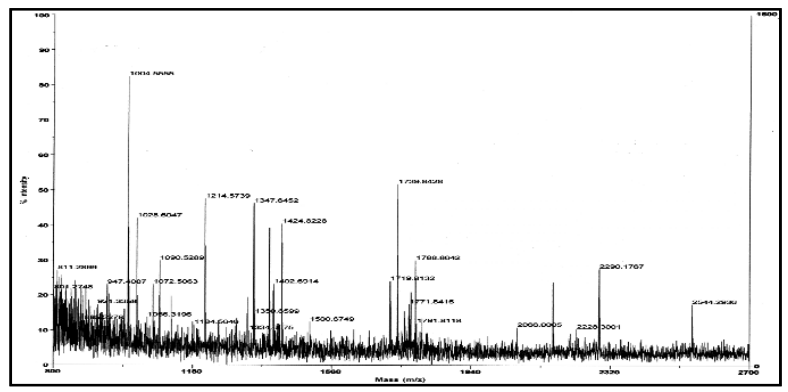

B

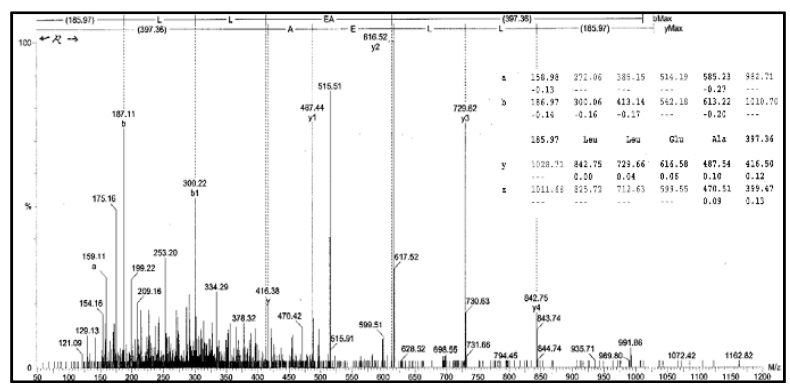

Figure 3. Identifying spot number 511 as Mn-SOD using MALDI-TOF (A) and ESI-TOF MS (B). 
A

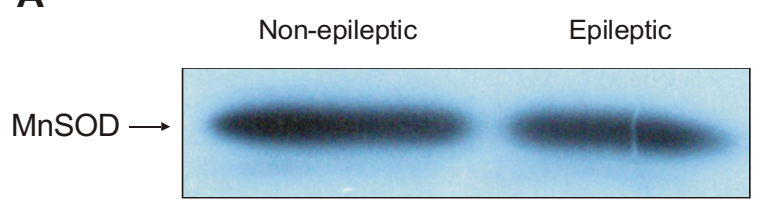

B

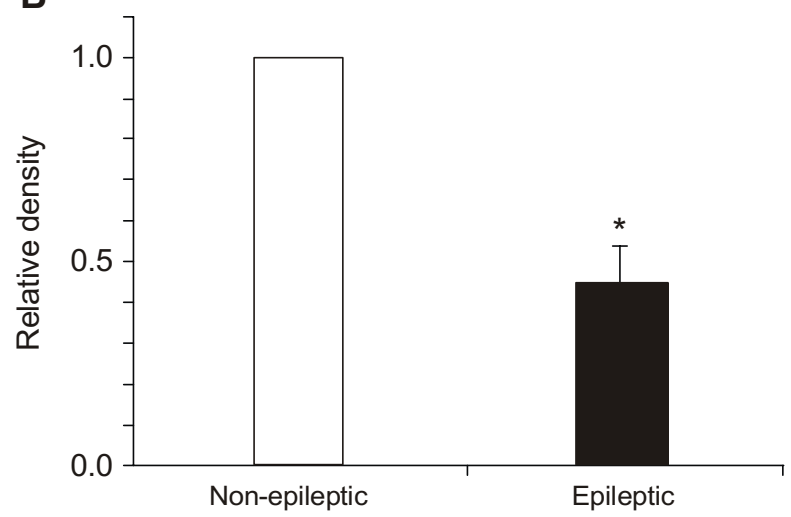

Figure 4. Western blot (A) and densitometric analysis (B) of Mn-SOD in cerebral cortexes from non-epileptic and temporal lobe epileptic patients. Results of densitometric analysis are expressed as mean \pm SEM from 12 experiments $\left({ }^{*} P<0.001\right)$.

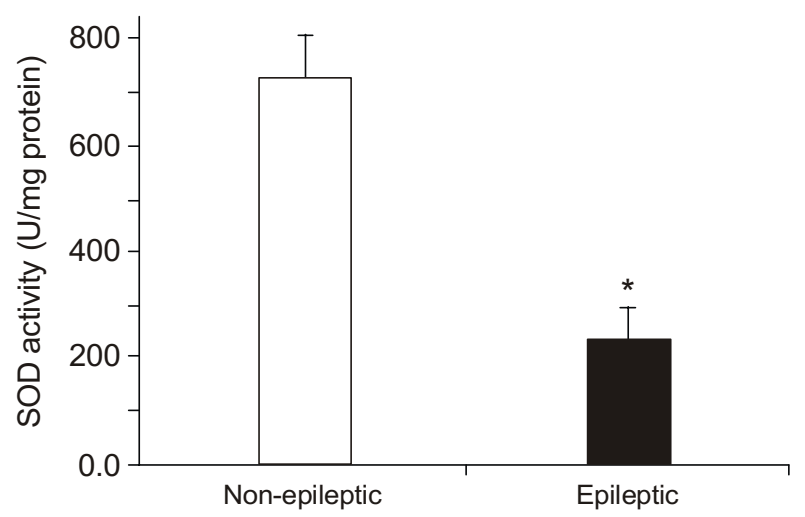

Figure 5. SOD activities of temporal lobe cortexes from non-epileptic and epileptic patients. Results of densitometric analysis are expressed as mean \pm SEM from 6 experiments $\left({ }^{*} P<0.001\right)$.

epileptic temporal neocortexes specimens when compared to the nonepileptic specimens. And the other four proteins were found to be expressed in the inverse manner. GPDH protein is one of the down-regulated ones in epileptic temporal neocortex (data not shown). The other proteins significantly different between temporal neocortexes from twelve nonepileptic and twelve epileptic subjects are UMP-CMP kinase, proteasome activator complex subunit 2, eukaryotic peptide chain release factor subunit 1 , apoptosis re-

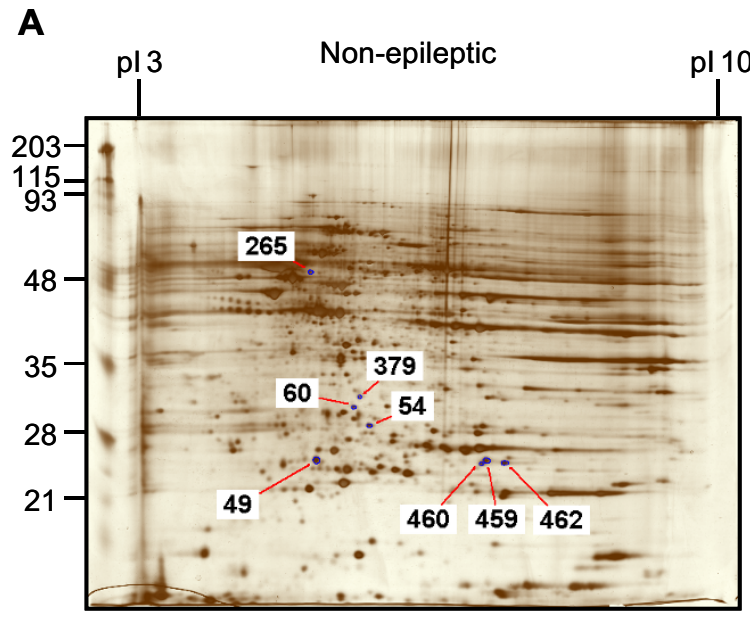

\section{B Epileptic}

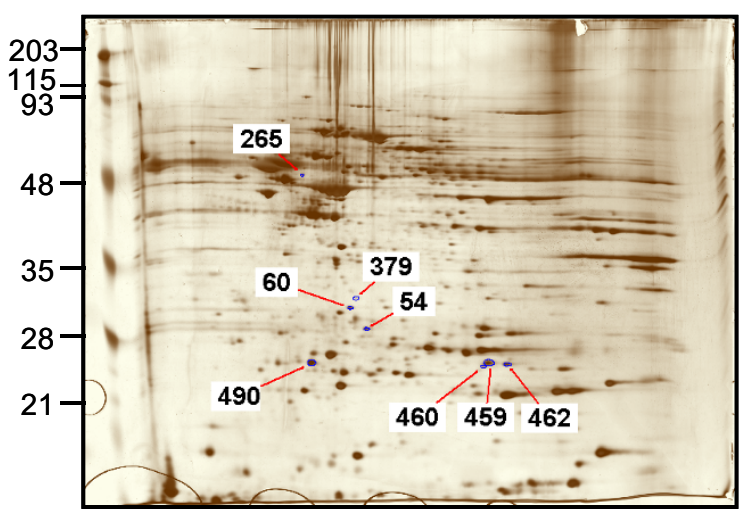

Figure 6. Representative 2-DE patterns of non-epileptic (A) and epileptic (B) human temporal lobes showing additional significant spots. Additional eight spots differentially expressed between the cerebral cortexes of non-epileptic (A) and epileptic (B) human temporal lobes. The gels were visualized with silver staining.

gulator BAX, cytoplasmic isoform beta, carbonic anhydrase VII, and DNA primase large subunit (polypeptide 2A, p58).

\section{Discussion}

Proteonomic methodology has emerged as most sensitive detectors of gene alterations and aberrant gene expression, protein modification as results of physio-pathogenesis. In the present study, proteonomic methods were employed to detect possible changes in protein expression in epileptic tissue. Nine proteins were identified to be significantly different between temporal neocortexes from twelve nonepileptic and twelve epileptic subjects. Furthermore, Mn-SOD was definitively identified as one of the proteins effected under epilepsy. 
Table 1. Other proteins significantly changed in temporal lobe from epileptic patients.

\begin{tabular}{rlcccc}
$\begin{array}{c}\text { Spot } \\
\text { no. }\end{array}$ & \multicolumn{1}{c}{ Protein } & $\begin{array}{c}\text { Accession } \\
\text { no. }\end{array}$ & $\begin{array}{c}\text { Measured } \\
\text { pl/mass }\end{array}$ & $\begin{array}{c}\text { pl/MW } \\
\text { (in database) }\end{array}$ & $\begin{array}{c}\text { Changes under } \\
\text { epilepsy }\end{array}$ \\
\hline 49 & UMP-CMP kinase & P30085 & $5.2 / 25.0$ & $5.4 / 22.2$ & Increase \\
54 & Proteasome activator complex subunit 2 & Q9UL46 & $5.8 / 29.0$ & $5.4 / 27.4$ & Increase \\
60 & Glyceraldehyde 3-phosphate dehydrogenase, Muscle & P00354 & $5.6 / 31.0$ & $6.6 / 35.9$ & Increase \\
265 & Eukaryotic peptide chain release factor subunit 1 (eRF 1) & P46055 & $5.1 / 46.0$ & $5.5 / 49.0$ & decrease \\
379 & Glycerol-3-phosphate dehydrogenase[NAD ${ }^{+}$],cytoplasmic & P21695 & $5.7 / 32.0$ & $5.8 / 37.6$ & decrease \\
459 & Apoptosis regulator BAX, cytoplasmic isoform beta & Q07814 & $7.3 / 26.0$ & $7.7 / 24.2$ & Increase \\
460 & Carbonic anhydrase VII & P43166 & $7.2 / 25.0$ & $6.9 / 29.7$ & decrease \\
462 & DNA primase larqe subunit (polvpeptide 2A, p58) & 10732519 & $7.5 / 25.0$ & $7.0 / 22.7$ & decrease \\
\hline
\end{tabular}

Oxidant stresses are known to induce tissue damage as well as neurological pathogenesis. Recurrent seizures increase the content of free radicals such as superoxide anion (Armstead et al., 1989). Systemic administration of kainate in rats increased mitochondrial superoxide production in the hippocampus at times preceding neuronal death (Liang et al., 2000). Free radicals are involved in pathogenesis of various diseases including arrythmia, cancer, atherosclerosis, and stroke (Halliwell and Gutteridge, 1999b; Kim et al., 2003). Free radical generation can induce seizure activity by inhibiting glutamine synthetase thereby increasing the level of an excitatory neurotransmitter glutamate (Oliver et al., 1990). There are, however, batteries of host defense mechanisms to neutralize such oxidant stresses. The biological antioxidant agents include SOD, catalase, glutathione peroxidase, thioredoxin and cytochrome c peroxidase (Halliwell and Gutteridge, 1999a).

There are several reports suggesting relationship between epilepsy and SOD. When epilepsy was induced in rats by systemic administration of pilocarpine, the total SOD activity was decreased by 25-35 $\%$ and the glutathion peroxidase activity increased by $39 \%$ in the hippocampus (Bellissimo et al., 2001). However they did not know whether the decrease of SOD activity was due to any changes of protein level or regulation of enzyme activity. The epileptic mutant EL mice had abnormally low levels of SOD isoenzyme activity in the hippocampal area (Murashima et al., 1998). Electroconvulsive shock-induced seizures in rats decreased superoxide dismutase (SOD) and glutathione peroxidase (GPX) activities in various brain regions (Erakovic et al., 2000). Erythrocyte CuZn-SOD was significantly lower in the progressive myoclonic epilepsies patients compared to controls (Ben-Menachem et al., 2000). On the other hand, when the amygdala of rats were stimulated electrically to induce epilepsy the whole brain SOD activity was increased by 2 -fold, and pretreatment of rats with SOD in- creased the threshold of epilepsy induction (Mori and Yokoyama, 1993).

Three types of SOD have been known: SOD1 (cytoplasmic Cu, Zn-SOD), SOD2 (mitochondrial MnSOD), SOD3 (extracellular Cu, Zn-SOD). In the present study, we found that the protein expression level of $\mathrm{Mn}-\mathrm{SOD}$ as well as the SOD activity were decreased clearly in epileptic temporal neocortex compared with those of nonepileptic temporal neocortex. These results demonstrate a possible link between epilepsy and Mn-SOD in human brain. Interestingly, there is a report that kainate-induced increases of mitochondrial superoxide production and hippocampal neuronal loss were attenuated in transgenic mice overexpressing Mn-SOD (Liang et al., 2000). Considering all of these, the increased accumulation of superoxide induced by the decreased activity of $\mathrm{Mn}-\mathrm{SOD}$ in epileptic cerebral cortex might contribute to the neuronal depolarization or hyperexcitability, indicating that Mn-SOD might be implicated with the pathogenesis of epilepsy. And it may be candidates as new therapeutic targets of epilepsy. However, further studies are needed to make it clear how the decreased Mn-SOD activity leads to development of epilepsy.

The other eight proteins identified in our proteomic analysis of epileptic temporal neocortexes might be involved in the pathogenesis of epilepsy and may be candidates as new therapeutic targets of epilepsy or early detection biomarkers.

Glycerol phosphate dehydrogenase (GPDH) is a soluble, multifunctional $\mathrm{NAD}^{+}$-linked enzyme associated with the metabolsm of triglycerides. It catalyzed the reversible reduction of dihydroxyacetone phosphate to glycerol phosphate, the precursor for phospholipids and triglycerides that is used for the biosynthesis of membrane components (Lin, 1977). In our proteomic analysis, GPDH protein is down-regulated in epileptic temporal neocortex. Finding of Link et al. (2000) showing the induction of GPDH in 
rat brain following seizure activity does support a role of GPDH participating in neuroprotective process as adaptive/repair response to components of stress after seizure. Thus, it may be hypothesized that the decrease of the neuroprotective effect by GPDH might contribute to the development of epilepsy. However, to clarify the pathophysiological role of GPDH in epilepsy, further studies are in order. The other proteins significantly different between temporal neocortexes from twelve nonepileptic and twelve epileptic subjects are UMP-CMP kinase, proteasome activator complex subunit 2, eukaryotic peptide chain release factor subunit 1, apoptosis regulator BAX, cytoplasmic isoform beta, carbonic anhydrase VII, and DNA primase large subunit (polypeptide 2A, p58). This is the first report describing the relationship between these proteins and epilepsy, although a lot of works are needed to understand the pathophysiological roles of these proteins.

The information obtained with this proteomic analysis will be very useful in understanding the pathogenesis of epilepsy as well as in finding candidates as new therapeutic targets or early detection biomarkers of epilepsy.

\section{Acknowledgement}

This work was supported by a grant from Chonbuk National University Hospital. We thank Korea Basic Science Institute (Daejeon, South Korea) for technical services of MALD-TOF and ESI-Q-TOF.

\section{References}

Armstead WM, Mirro R, Leffler CW, Busija DW. Cerebral superoxide anion generation during seizures in newborn pigs. J Cereb Blood Flow Metab 1989;9:175-9

Bellissimo MI, Amado D, Abdalla DS, Ferreira EC, Cavalheiro EA, Naffah-Mazzacoratti MG. Superoxide dismutase, glutathione peroxidase activities and the hydroperoxide concentration are modified in the hippocampus of epileptic rats. Epilepsy Res 2001;46:121-8

Ben-Menachem E, Kyllerman M, Marklund S. Superoxide dismutase and glutathione peroxidase function in progressive myoclonus epilepsies. Epilepsy Res 2000;40:33-9

Erakovic V, Zupan G, Varljen J, Radosevic S, Simonic A. Electroconvulsive shock in rats: changes in superoxide dismutase and glutathione peroxidase activity. Brain Res Mol Brain Res 2000;76:266-74

Halliwell B, Gutteridge JMC. Antioxidant defences. In Free Radicals in Biology and Medicine 1999a, 105-245, Oxford University Press, New York

Halliwell B, Gutteridge JMC. Free radicals, other reactive species and disease. In Free Radicals in Biology and Medi- cine 1999b, 617-783, Oxford University Press, New York

Hellman U, Wernstedt C, Gonez J, Heldin CH. Improvement of an "In-Gel" digestion procedure for the micropreparation of internal protein fragments for amino acid sequencing. Anal Biochem 1995;224:451-5

Kim YH, Lee JH, Lim do S, Shim WJ, Ro YM, Park GH, Becker KG, Cho-Chung YS, Kim MK. Gene expression profiling of oxidative stress on atrial fibrillation in humans. Exp Mol Med. 2003;35:336-49

Lai JCK, Clark JB. Preparation of synaptic and nonsynaptic mitochondria from mammalian brain. In Methods in Enzymology (Fleischer S, Packer L. eds), 1979, 51-60, Academic Press, New York, NY

Liang LP, Ho YS, Patel M. Mitochondrial superoxide production in kainate-induced hippocampal damage. Neuroscience 2000;101:563-70

Link WA, Kauselmann G, Mellstrom B, Kuhl D, Naranjo JR. Induction of glycerol phosphate dehydrogenase gene expression during seizure and analgesia. J Neurochem 2000; $75: 1419-28$

Meisler MH, Kearney J, Ottman R, Escayg A. Identification of epilepsy genes in human and mouse. Annu Rev Genet 2001;35:567-88

Mori N, Yokoyama H. Role of superoxide dismutase in a kindling model of epilepsy. Comp Biochem Physiol C 1993; 104:373-6

Murashima YL, Kasamo K, Suzuki J. Antiepileptic effects of allopurinol on EL mice are associated with changes in SOD isoenzyme activities. Epilepsy Res 1998;32:254-65

Nebot C, Moutet M, Huet P, Xu JZ, Yadan JC, Chaudiere $J$. Spectrophotometric assay of superoxide dismutase activity based on the activated autoxidation of a tetracyclic catechol. Anal Biochem 1993;214:442-51

Oliver CN, Starke-Reed PE, Stadtman ER, Liu GJ, Carney JM, Floyd RA. Oxidative damage to brain proteins, loss of glutamine synthetase activity, and production of free radicals during ischemia/reperfusion- induced injury to gerbil brain. Proc Natl Acad Sci USA 1990;87:5144-7

Park SA, Kim TS, Choi KS, Park HJ, Heo K, Lee BI. Chronic activation of CREB and p90RSK in human epileptic hippocampus. Exp Mol Med. 2003;35:365-70

Patel MN. Oxidative stress, mitochondrial dysfunction, and epilepsy. Free Radic Res 2002;36:1139-46

Scheler C, Lamer S, Pan Z, Li XP, Salnikow J, Jungblut $P$. Peptide mass fingerprint sequence coverage from differently stained proteins on two-dimensional electrophoresis patterns by matrix assisted laser desorption/ionization-mass spectrometry (MALDI-MS). Electrophoresis 1998;19:918-27

Shevchenko A, Wilm M, Vorm O, Mann M. Mass spectrometric sequencing of proteins silver-stained polyacrylamide gels. Anal Chem 1996;68:850-8

Stefan H, Halasz P, Gil-Nagel A, Shorvon S, Bauer G, Ben-Menachem E, Perucca E, Wieser HG, Steinlein $O$. Recent advances in the diagnosis and treatment of epilepsy. Eur J Neurol 2001;8:519-39 